\title{
BMJ Open A publicly well-accepted measure versus an academically desirable measure of health inequality: cross- sectional comparison of the difference between income quintiles with the slope index of inequality
}

\author{
Young-Ho Khang, ${ }^{\circledR 1,2}$ Dohee Lim, ${ }^{\circledR 2}$ Jinwook Bahk, ${ }^{3}$ Ikhan Kim, ${ }^{\circledR 1}$ \\ Hee-Yeon Kang, ${ }^{1}$ Youngs Chang, ${ }^{1}$ Kyunghee Jung-Choi ${ }^{4}$
}

To cite: Khang Y-H, Lim D, Bahk J, et al. A publicly wellaccepted measure versus an academically desirable measure of health inequality: cross-sectional comparison of the difference between income quintiles with the slope index of inequality. BMJ Open 2019;9:e028687. doi:10.1136/ bmjopen-2018-028687

- Prepublication history and additional material for this paper are available online. To view these files, please visit the journal online (http://dx.doi. org/10.1136/bmjopen-2018028687).

Received 21 December 2018 Revised 29 May 2019 Accepted 7 June 2019

Check for updates

(c) Author(s) (or their employer(s)) 2019. Re-use permitted under CC BY-NC. No commercial re-use. See rights and permissions. Published by BMJ.

For numbered affiliations see end of article.

Correspondence to Professor Young-Ho Khang; yhkhang@snu.ac.kr

\section{ABSTRACT}

Objectives The difference between income quintiles in health is relatively well accepted by the general public as a measure of health inequality. However, the slope index of inequality (SII) in health reflects the patterns of all social groups, including the middle $60 \%$, and it could therefore be considered more academically desirable. If these two measures are closely correlated, the widespread use of the difference between income quintiles in health would be better supported. This study was conducted to compare differences between income quintiles in life expectancy (LE) and healthy life expectancy (HLE) with the SII.

Design Cross-sectional comparison using correlational analysis of district level income differences in LE and HLE with associated SII.

Setting All 252 subnational districts of Korea.

Participants A total of 342439895 subjects (171 287

729 men, 171152166 women) and 1753476 deaths

(970928 men, 782548 women) between 2008 and 2014 were analysed.

Primary and secondary outcome measures Difference in LE and HLE by income quintile and associated SII. Results The Pearson correlation coefficients between differences between income quintiles and the SII were generally high: $0.97(95 \% \mathrm{Cl} 0.96$ to 0.98$)$ for $\mathrm{LE}$ in men and women combined and $0.96(95 \% \mathrm{Cl} 0.94$ to 0.97$)$ for HLE in men and women combined. In most districts, the SII was greater than the difference between income quintiles. Conclusion Differences between income quintiles were closely correlated with the SII. The widespread use of differences between income quintiles in health as a measure of health inequality may be preferable for communicating results of health inequality measurements to the public.

\section{INTRODUCTION}

Various measures of socioeconomic health inequalities have been used. ${ }^{1-5}$ Differences between income quintiles in health are relatively well accepted by the general public.

\section{Strengths and limitations of this study}

- This study employed total Korean population and mortality data and showed income-based inequalities in life expectancy (LE) in all 252 districts of Korea.

- This study presented inequalities in healthy life expectancy (HLE) by income quintile at the subnational district level in Korea.

- This study suggests that the widespread use of differences between income quintiles in LE and HLE as a measure of health inequality may be preferable for communicating results of health inequality measurements to the public.

- The findings may not be true for other socioeconomic position indicators (eg, education) or other health outcomes than LE and HLE.

The general public is accustomed to the $20: 20$ or 20/20 ratio, in which the top $20 \%$ of a population in terms of income distribution is compared with the bottom $20 \%$. However, the use of differences between income quintiles in health could be criticised because this metric does not reflect the patterns of health status in the middle $60 \%$, and thus obscures the overall patterns of inequalities. The slope index of inequality (SII) in health reflects the patterns of all social groups, and could therefore be considered academically desirable..$^{2-4}$ To calculate the correspondence of the SII to differences between income quintiles, a relative income quintile indicator (a value between 0 and 1 ) reflecting the relative position in the cumulative population distribution of the central subject in the income hierarchy should be employed. For example, if the lowest income quintile reflects the bottom 


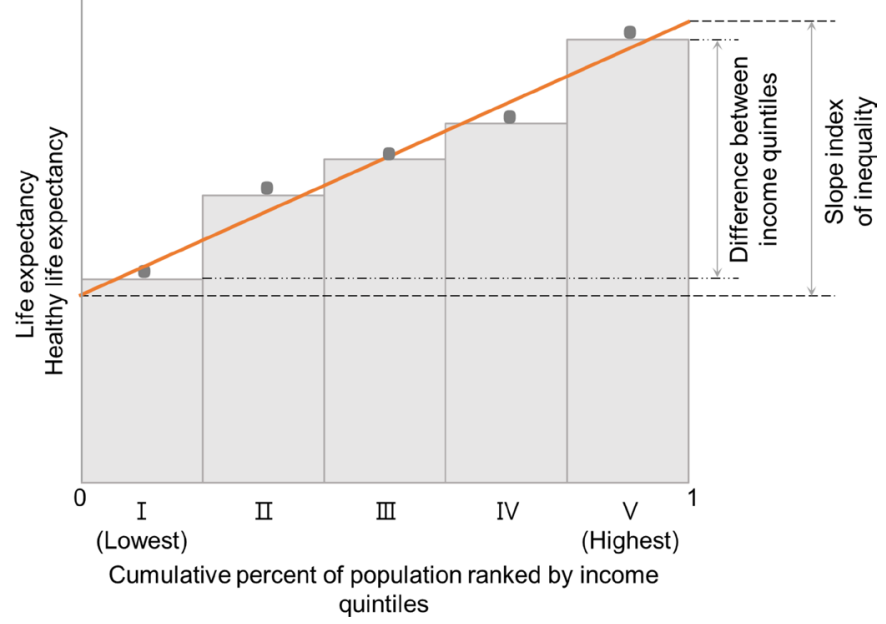

Figure 1 Hypothetical example of the difference between income quintiles in life expectancy and healthy life expectancy and the associated slope index of inequality.

$20 \%$ of the population, that group is assigned a relative rank of $0.10\left(0+0.5^{*} 0.20\right)$. The relative position indicators are used as an independent variable in the linear regression analysis for SII, as shown in figure 1 . Thus, the SII in life expectancy (LE) and healthy life expectancy (HLE) reflects the absolute difference in LE and HLE between the lowest end of the income hierarchy and the very top income level (figure 1). Although the health status of each social group and the SII in health both provide valuable information on socioeconomic health inequalities, these metrics might not be successful in delivering simple and easily understandable messages to the public. Therefore, differences between income quintiles in health could be preferable as a way to communicate information about health inequalities to the general public.

In South Korea (hereafter 'Korea'), differences between income quintiles have been widely accepted in the media, ${ }^{67}$ and have been used to suggest national targets for equity in health. ${ }^{89}$ For instance, differences between income quintiles in the mortality rate and prevalence of various health behaviours were suggested as national targets for health equity in the National Health Plan 2010 in Korea. ${ }^{8}$ Differences between income quintiles in LE and HLE were also presented as a candidate overarching goal for health equity in the upcoming National Health Plan 2030. ${ }^{10}{ }^{11}$ In addition, ahead of the local elections that took place nationwide in mid-2018, the Korea Society of Equity in Health publicly released health gap profiles for all 252 districts in Korea to boost the public's and politicians' awareness of health inequality (see online supplementary figure 1) ${ }^{6712}$ The profile for each district presented its ranking in terms of differences between income quintiles in LE and HLE. ${ }^{12}$ However, the question arises of whether the ranking based on differences between income quintiles corresponds to the ranking based on the SII. If these two measures are closely correlated, the widespread use of differences between income quintiles in health would be better academically supported. If not, the use of differences between income quintiles in health, especially for comparing the magnitude of socioeconomic health inequalities, should be reconsidered. To the best of our knowledge, no prior studies examined the correlation between SII in LE and HLE and differences between income quintiles. This study was conducted to compare differences between income quintiles in LE and HLE with the SII using subnational data from all 252 districts of Korea.

\section{METHODS \\ Data}

Data on LE and HLE by income quintile were derived from the health gap profiles published by the Korean Society for Equity in Health (online supplementary figure 1). ${ }^{12}$ These profiles were generated based on a prior study conducted by the authors of this paper. ${ }^{11}$ In that study, we constructed life tables using the National Health Information Database (NHID) of the National Health Insurance Service, ${ }^{13}$ which was individually linked to death certificate data from Statistics Korea. A prior study showed that the NHID could be used for monitoring LE and LE inequalities at the district level. ${ }^{14}$ Combined data from 2010 to 2015 on population and deaths according to sex, 5-year age-specific groups $(<1,1-4,5-9, \ldots$, and $\geq 85$ years) and income quintiles for 252 districts were used to ensure stable mortality figures at the district level. ${ }^{15-17}$ The life tables were created using the annual population of national health insurance beneficiaries (except for foreigners and soldiers) and the number of deaths registered in each calendar year. A total of 295459279 subjects (147 668147 men, 147791132 women) and 1542884 deaths (850003 men, 692881 women) were analysed. The Kannisto-Thatcher method was employed to expand the open-ended age interval $85+$ to estimate the probability of dying for each of the 5-year age groups $85-89,90-94, \ldots, 120-124$, and $125+{ }^{18}$

For HLE, we employed the Korea Community Health Survey (KCHS), which is a nationwide, community-based, cross-sectional survey that has been conducted since 2008 to produce comparable health statistics at the district level in Korea. ${ }^{19}$ Self-rated poor health was used as the morbidity indicator for the computation of HLE. Combined data from 2008 through 2014 on total subjects and self-rated poor health were used to ensure stable estimations of self-rated poor health prevalence according to sex, 5-year age-specific group (20-24, 25-29, ..., and $\geq 80$ years) and income quintile for 252 districts. At the time of the study, the $2015 \mathrm{KCHS}$ data were not available. Incomebased inequality in self-rated poor health at the district level has been investigated in a prior Korean study. ${ }^{20}$ Since the KCHS is a survey of people aged 19 and over, for those younger than 20 years, the self-rated poor health of the 20-24 years old age group was used instead. This study included data from the KCHS between 2008 and 2014 for a total of 1577541 respondents over 20 years of age. Mortality data for the same 7-year period from the NHID 
were employed. For HLE, a total of 342439895 subjects (171 287729 men, 171152166 women) and 1753476 deaths (970928 men, 782548 women) between 2008 and 2014 were analysed. Based on the calculated mortality rate and prevalence of self-rated poor health, HLE was estimated with the Sullivan method. ${ }^{21}$

\section{Socioeconomic position indicator}

The national health insurance contributions contained in the NHID were used as a proxy for income. Prior Korean studies have revealed that the national health insurance contributions levied on salaries and household assets (car ownership, housing, etc.) were closely associated with mortality and LE. ${ }^{22-24}$ In this study, we took into account household size and generated equivalised health insurance contributions. In the KCHS, income data were based on self-reported information on monthly or yearly household income, which was collected as a continuous variable between 2008 and 2013 and as a categorical variable in 2014 . The categorical variable of income in 2014 was converted to a continuous variable by taking the median value of each category. Since the KCHS data were combined in 2008-2014, the household income for each year was adjusted using the consumer price index to account for fluctuations in inflation during the study period. For 110579 (7.0\%) KCHS participants with a missing household income, the value for income was replaced by multiple imputation using a propensity score generated from age, sex, household size, occupation and educational attainment of the respondents. Prior Korean studies have employed the above-described approach to construct the income quintile variable. $^{2025}$ Thus, in this study, each income group in each district accounted for approximately $20 \%$ of total population in a district.

\section{Statistical analysis}

Based on data on LE and HLE by income quintile for each district in Korea, we estimated the SII in LE and HLE using linear regression analyses. We calculated the mean, SD and range of differences between income quintiles and the associated SII for both LE and HLE in 252 districts of Korea (table 1). We then created scatter plots of the relationship between differences between income quintiles and the SII in LE and HLE and conducted correlation analyses of this relationship (figure 2). Finally, to identify the characteristics of the districts where the SII in LE and HLE was smaller than the difference between income quintiles, we compared the numbers of population and deaths between districts where the SII was greater than or equal to the difference between income quintiles and districts where the SII was less than the difference between income quintiles (table 2).

\section{Patient and public involvement}

No patients were involved in the development and design of this study.

Table 1 Mean, SD and range of differences between income quintiles and the slope index of inequality in life expectancy and healthy life expectancy among 252 districts in Korea

$\begin{array}{llll}\text { Mean } & \text { SD } & \text { Min } & \text { Max }\end{array}$

Life expectancy in 2010-2015

Men and women combined

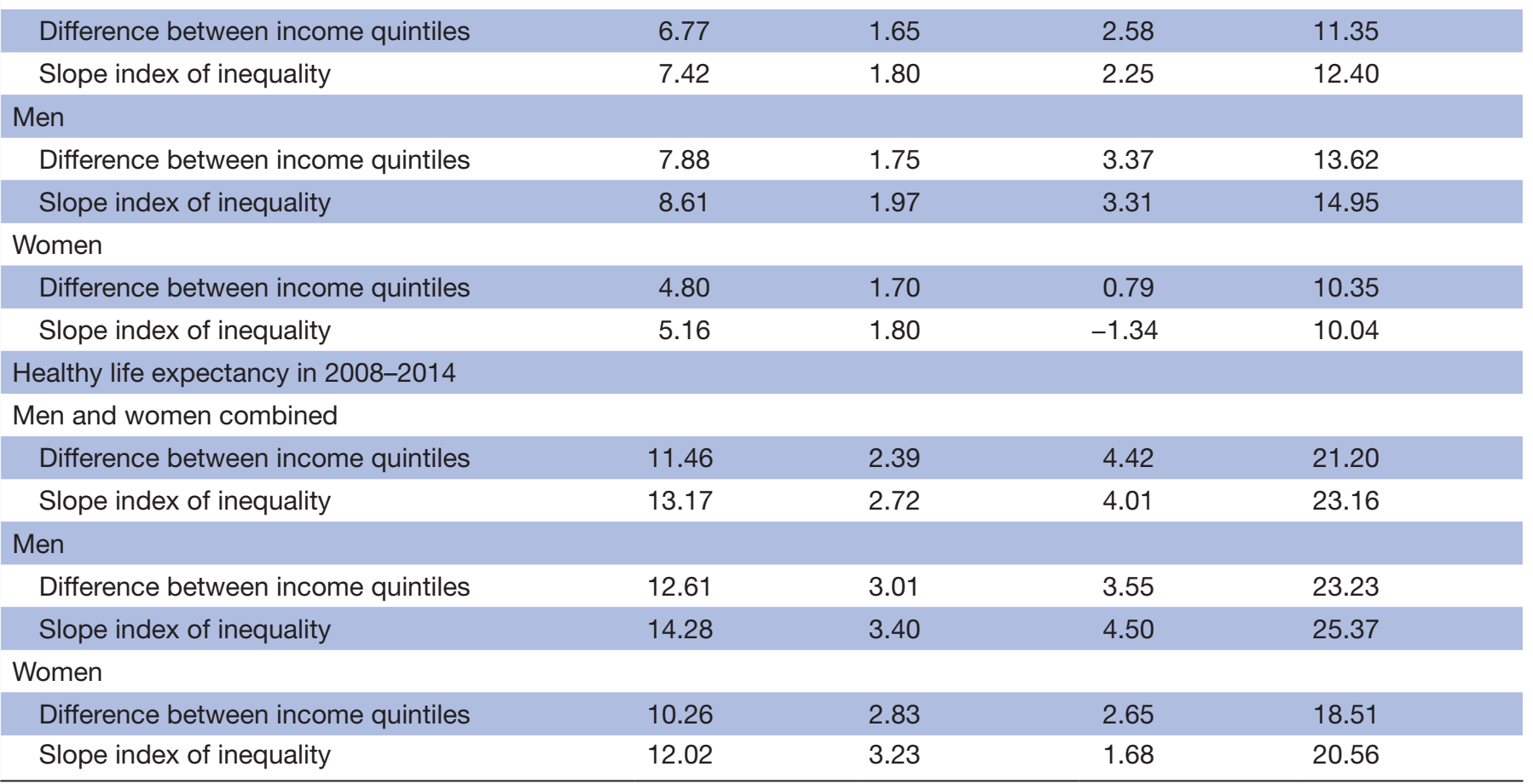


(A)

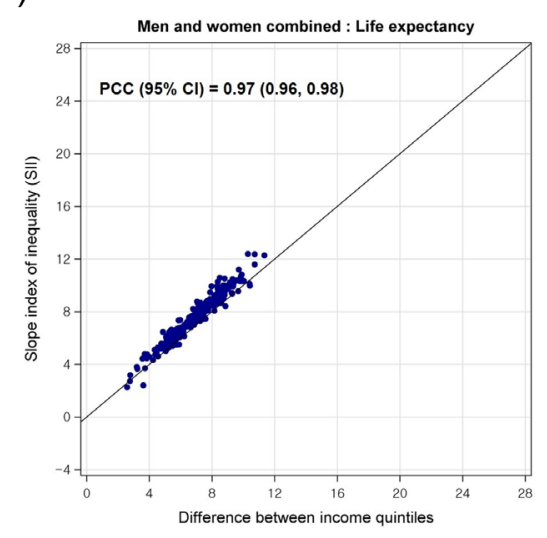

(B)

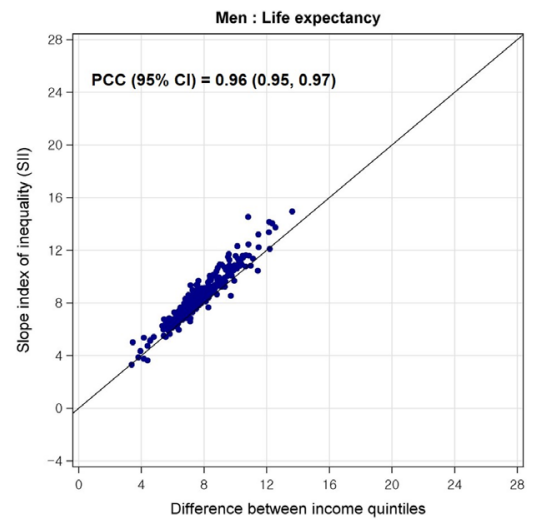

(C)

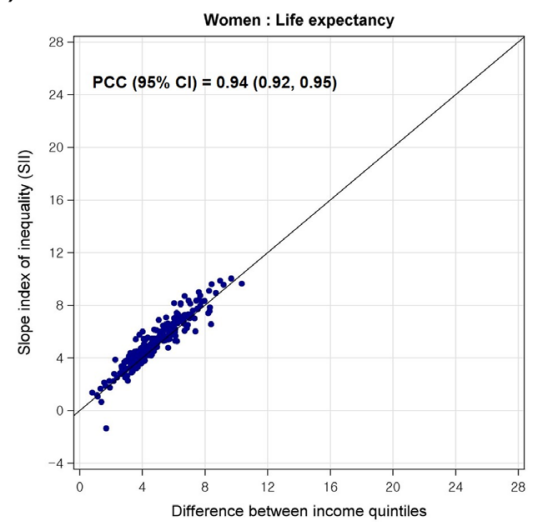

(D)

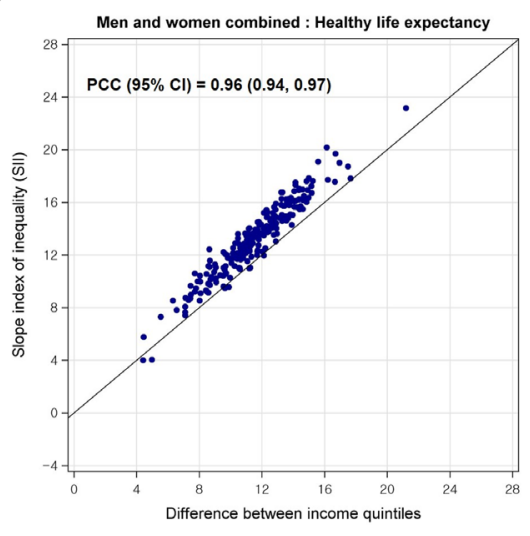

(E)

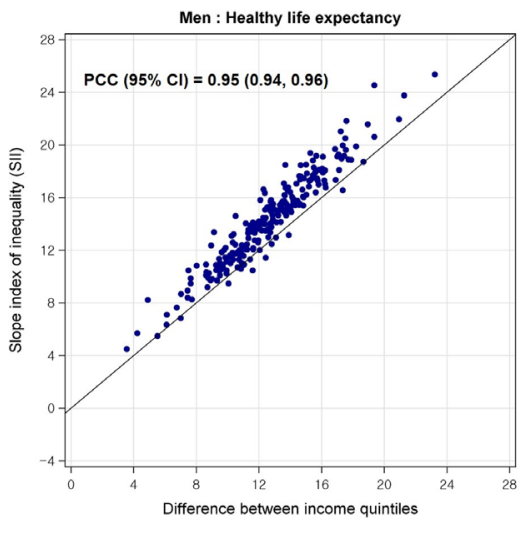

$(\mathrm{F})$

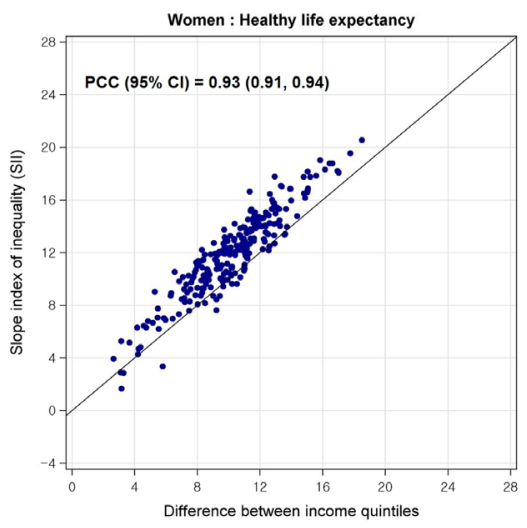

Figure 2 Scatter plots and Pearson correlation coefficients (PCCs) $(95 \%$ Cls) for the relationship between the difference between income quintiles and the slope index of inequality (SII) in life expectancy (2010-2015) and healthy life expectancy (2008-2014) among 252 districts of Korea.

\section{RESULTS}

Table 1 shows measures of central tendency (mean) and dispersion (SD and range) for the difference between income quintiles and the SII with regard to LE and HLE at the district level. The magnitudes of inequality were greater for HLE than for LE. Although men had greater inequalities by income than women in both LE and HLE, the sex difference in LE inequality among income quintiles (a 7.88-year difference in men vs a 4.80-year difference in women) was greater than the sex difference in HLE inequality among income quintiles (a 12.61-year difference in men vs a 10.26-year difference in women). The measures of dispersion indicated that the magnitudes of inequality in LE and HLE varied more in men 
Table 2 Comparison of mean (SD) annual numbers of population and deaths between districts where the slope index of inequality (SII) was greater than or equal to the difference between income quintiles (DIQ) in life expectancy and healthy life expectancy and districts where the SII was less than the DIQ among 252 districts in Korea

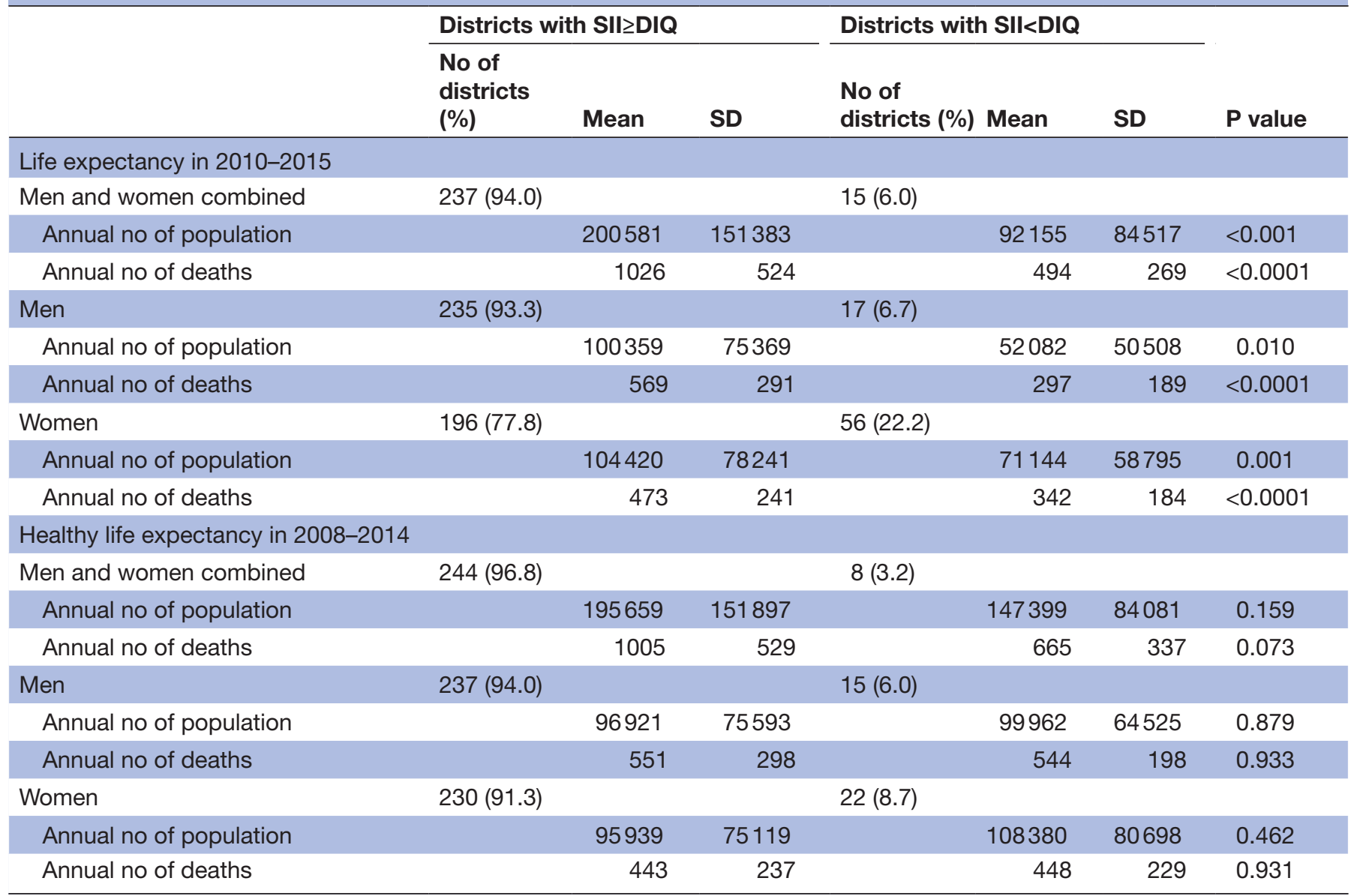

than in women. Table 1 also shows that the magnitude of inequality measured by the SII was generally greater than that measured by the difference between income quintiles in both LE and HLE.

Figure 2 shows correlations of differences between income quintiles in LE and HLE with the SII. The correlation coefficients were generally high: 0.97 (95\% CI 0.96 to $0.98)$ for LE in men and women combined and $0.96(95 \%$ CI 0.94 to 0.97 ) for HLE in men and women combined. The lowest correlation coefficient was 0.93 (95\% CI 0.91 to 0.94 ) for HLE in women. In most of the districts, the SII was greater than the difference between income quintiles. Only one island district with the smallest population (annual population: 5199 men and 4789 women) of all 252 districts, Ulleung-gun, showed a negative SII (-1.34 years) for LE among women because of the small numbers of deaths in the middle $60 \%$ of the income distribution (annual number of female deaths in Ulleung-gun: 29).

Table 2 presents the number of districts where the SII was lower than the difference between income quintiles (located at the lower part of diagonal line in figure 1). For HLE in men and women combined, 8 such districts (3.2\% of the total 252 districts) were found, whereas 56 districts $(22.2 \%$ of total 252 districts) showed this pattern for LE in women. It was more common for the SII to be smaller than the difference between income quintiles for LE than for HLE. For LE, the districts located at the lower part of the diagonal line in figure 1 recorded significantly smaller district populations and significantly fewer deaths than the districts at the upper part of the diagonal line in figure 1 . However, this was not true for HLE.

\section{DISCUSSION}

This study examined the relationship of differences between income quintiles in LE and HLE with the corresponding SII. The results showed that the two measures were closely correlated at the subnational district level when we compared income-based inequalities in LE and HLE. The Pearson correlation coefficients were about 0.93-0.97. We considered the difference between income quintiles to be a publicly well-accepted measure of health inequality, whereas the SII was considered to be an academically desirable measure. Although no survey has directly compared the public's understanding of these two measures, it would be reasonable to expect it to be difficult to deliver simple and self-evident messages to the public using information on a regression-based measure 
(slope index inequality). In contrast, the difference between income quintiles might be more easily understood by the public. The Korean experience in releasing health gap profiles containing the results of inequalities in LE and HLE between income quintiles confirmed that information on differences between income quintiles in LE and HLE was effectively received by the media. ${ }^{6} 712$ Therefore, the results of this study suggest that the difference between income quintiles in LE and HLE might be robust against serious academic criticisms regarding inequality patterns in the middle $60 \%$ of the income distribution, and could be more widely used for communicating results on health inequality measurements to the public.

When a stepwise pattern was found in the relationship between a socioeconomic position indicator and a health outcome, the magnitude of inequalities measured by SII tended to be greater than that measured by the difference between income quintiles (see figure 1). ${ }^{3}{ }^{4}$ This study showed that the mean values of the SII were greater than the differences between income quintiles (see table 1). Most districts had a greater magnitude of health inequalities as measured by the SII than by differences between income quintiles (the upper part of the diagonal line in figure 1). However, for LE in women, a substantial proportion of districts recorded smaller magnitudes of inequalities measured by SII. This could have occurred for two reasons. One factor may have been the presence of weaker associations between income and LE among women than among men, as indicated in this study (table 1). The other possible reason may be the relatively small numbers of district-level deaths among women (see table 2), resulting in unstable mortality rates in some age groups (especially in young groups). In Korea, it has been reported that pro-rich socioeconomic inequalities in selfrated poor health were evident in all districts. ${ }^{20}$ Since data on self-rated poor health from the KCHS were incorporated into the calculation of HLE, in addition to mortality data, the difference between income quintiles was higher than the SII for HLE in fewer districts than was the case for LE. The sex difference in HLE inequality by income was not as great as in the sex difference in LE inequality by income (see table 1 ). In addition, no significant differences in the population size and numbers of deaths were found for HLE between districts in the upper part of the diagonal line in figure 1 and districts in the lower part (see table 2). These findings suggest that, with sufficient population size and associated deaths, stepwise patterns of inequalities in LE by income are expected, resulting in the SII being greater than the difference between income quintiles. In addition, because of the two sources (mortality and self-rated health) that were used for health inequalities, a stepwise pattern in health inequalities was more clearly shown for HLE than for LE.

This study has certain limitations. First, this study only focused on SII and differences by income quintiles as health inequality measures, considering that SII is the summary index directly corresponding to the differences by income quintiles. Comparison with other measures including concentration index or other level-dependent indicators ${ }^{1-4}{ }^{26}$ may produce various estimates for correlation coefficients between measures. Second, the study period for LE and HLE was different. This was because at the time of the original investigation the 2015 KCHS data were not publicly available and because all available KCHS data since 2008 were necessary to obtain stable numbers of subjects for each district-specific, sex-specific, income-specific and age-specific group considering the small sample size of the KCHS for each year (a total of approximately 900 subjects) in each district. ${ }^{11}$ Third, this study employed midpoints of the income quintiles to calculate the SII. It was assumed that the SII would become greater than the difference between income quintiles if income and LE were linearly associated. However, this assumption may not be true for other socioeconomic position indicators, for which the population size varies across subcategories of the indicators. For example, in a society where the combination of low education (eg, primary or less education) with a very high mortality rate is extremely marginal (ie, the population size is quite small), the calculated SII might not be greater than the gaps between high-education and low-education groups. Fourth, this study only presented results on LE and HLE. A different result may be possible for other health indicators. However, when we replicated the analysis for self-rated poor health, a popular morbidity indicator, presented in a prior Korean study, ${ }^{20}$ we could obtain a similarly high level of correlations (correlation coefficients of 0.96-0.97) for the relationship of differences between income quintiles with SII in self-rated poor health (see online supplementary figure 2).

In conclusion, this study showed that differences between income quintiles in LE and HLE were closely correlated with the SII for LE and HLE. The widespread use of differences between income quintiles in health as a measure of health inequality may therefore be preferable for communicating results of health inequality measurements to the public.

\section{Author affiliations}

${ }^{1}$ Department of Health Policy and Management, Seoul National University College of Medicine, Seoul, Republic of Korea

${ }^{2}$ Institute of Health Policy and Management, Seoul National University Medical Research Center, Seoul, Republic of Korea

${ }^{3}$ Department of Public Health, Keimyung University, Daegu, Republic of Korea ${ }^{4}$ Department of Occupational and Environmental Medicine, Ewha Womans University School of Medicine, Seoul, Republic of Korea

Acknowledgements The authors would like to thank Yeon-Yong Kim and JongHeon Park at the Big Data Steering Department, the National Health Insurance Service for their support with data curation.

Contributors Y-HK: conceived the study, supervised the data analysis and drafted the manuscript. DL: computed slope index of inequality and contributed to drafting the manuscript. JB, IK, H-YK: contributed to the calculations of life expectancy and healthy life expectancy. All authors: read and approved the final manuscript.

Funding This research was supported by a grant of the Korea Health Technology R\&D Project through the Korea Health Industry Development Institute, funded by the Ministry of Health \& Welfare, Republic of Korea (grant number: HI18C0446). 
Competing interests None declared.

Patient consent for publication Not required.

Ethics approval The study was approved by the National Health Insurance Service of Korea and the Seoul National University Hospital Institutional Review Board (IRB No. E-1810-008-975).

Provenance and peer review Not commissioned; externally peer reviewed.

Data sharing statement Data are available in a public, open access repository.

Open access This is an open access article distributed in accordance with the Creative Commons Attribution Non Commercial (CC BY-NC 4.0) license, which permits others to distribute, remix, adapt, build upon this work non-commercially, and license their derivative works on different terms, provided the original work is properly cited, appropriate credit is given, any changes made indicated, and the use is non-commercial. See: http://creativecommons.org/licenses/by-nc/4.0/.

\section{REFERENCES}

1. Harper S, Lynch J. Health inequalities: measurement and decomposition. In: Oakes JM, Kaufman JS, eds. Methods in social epidemiology. 2nd edn. San Francisco, CA: Jossey-Bass \& Pfeiffer Imprint, Wiley, 2017:91-131.

2. Hosseinpoor A. Handbook on health inequality monitoring - with a special focus on low and middle-income countries. Geneva: World Health Organization, 2013.

3. Harper S, Lynch J. Measuring health inequalities. In: Oakes JM, Kaufman JS, eds. Methods in social epidemiology. San Francisco, CA: Jossey-Bass A Wiley Imprint, 2006:134-68.

4. Mackenbach JP, Kunst AE. Measuring the magnitude of socioeconomic inequalities in health: an overview of available measures illustrated with two examples from Europe. Soc Sci Med 1997;44:757-71.

5. Khang YH, Yun SC, Lynch JW. Monitoring trends in socioeconomic health inequalities: it matters how you measure. BMC Public Health 2008;8:66.

6. Yonhap News. Upper income $20 \%$ live longer and healthier everywhere in Korea. Serious health inequalities exist. http://www. yonhapnews.co.kr/bulletin/2018/03/26/0200000000AKR2018032 6106300017.HTML (Accessed 07 Nov 2018).

7. Joongang ilbo. The rich live longer and healthier. A 13-year difference in health expectancy. https://news.joins.com/article/22476822 (Accessed 07 Nov 2018).

8. Ministry of Health and Welfare, Korea Institute for Health and Social Affairs. Establish of new health plan 2010. Seoul: Ministry of Health and Welfare and Korea Institute for Health and Social Affairs, 2005:42-6.

9. Khang YH, Lee SI. Health inequalities policy in Korea: current status and future challenges. J Korean Med Sci 2012;27:S33-40.

10. Korean Society for Equity in Health, Ministry of Health and Welfare of Korea. Developing key indicators of health equity and strategies for reducing health disparity in national health plan. Sejong, Korea:
Korean Society for Equity in Health and Ministry of Health and Welfare of Korea, 2016.

11. Khang YH, Yun SC, Bahk J, et al. Developing indicators for evaluating and monitoring the health plan 2020 using the National Health Information Database of the National Health Insurance Service in Korea. Seoul, Korea: National Health Insurance Service of Korea, Seoul National University, 2016.

12. Korean Society for Equity in Health. Health gap profiles at the national, provincial, district levels. https://drive.google.com/open?id= 1Czdweu2 Db5QsKJOiqSGo1BJp2ody0nl (Accessed 07 Nov 2018).

13. Cheol Seong S, Kim YY, Khang YH, et al. Data Resource Profile: The National Health Information Database of the National Health Insurance Service in South Korea. Int J Epidemiol 2017;46:799-800.

14. Bahk J, Kim YY, Kang HY, et al. Using the National Health Information Database of the National Health Insurance Service in Korea for Monitoring Mortality and Life Expectancy at National and Local Levels. J Korean Med Sci 2017;32:1764-70.

15. Eayres D, Williams ES. Evaluation of methodologies for small area life expectancy estimation. J Epidemiol Community Health 2004;58:243-9.

16. Jonker MF, van Lenthe FJ, Congdon PD, et al. Comparison of Bayesian random-effects and traditional life expectancy estimations in small-area applications. Am J Epidemiol 2012;176:929-37.

17. Scherbov S, Ediev D. Significance of life table estimates for small populations: Simulation-based study of estimation errors. Demogr Res 2011;24:527-50.

18. Thatcher AR, Vi K, Vaupel JW. The force of mortality at ages 80 to 120. Odense, Denmark: Odense University Press, 1998.

19. Kang YW, Ko YS, Kim YJ, et al. Korea community health survey data profiles. Osong Public Health Res Perspect 2015;6:211-7.

20. Kim I, Bahk J, Yun SC, et al. Income gaps in self-rated poor health and its association with life expectancy in 245 districts of Korea. Epidemiol Health 2017;39:e2017011.

21. Jagger C, Hauet E, Brouard N. Health expectancy calculation by the Sullivan method: a practical guide. Tokyo: Nihon University, Population Research Institute, 1999.

22. Jung-Choi K, Khang $\mathrm{YH}$, Cho HJ. Socioeconomic differentials in cause-specific mortality among 1.4 million South Korean public servants and their dependents. J Epidemiol Community Health 2011;65:632-8.

23. Khang YH, Bahk J, Yi N, et al. Age- and cause-specific contributions to income difference in life expectancy at birth: findings from nationally representative data on one million South Koreans. Eur J Public Health 2016;26:242-8.

24. Khang YH, Yang S, Cho HJ, et al. Decomposition of socio-economic differences in life expectancy at birth by age and cause of death among 4 million South Korean public servants and their dependents. Int J Epidemiol 2010;39:1656-66.

25. Kim I, Bahk J, Yoon TH, et al. Income differences in smoking prevalences in 245 districts of South Korea: patterns by area deprivation and urbanity, 2008-2014. J Prev Med Public Health 2017;50:100-26.

26. Erreygers G, Kessels R. Socioeconomic status and health: a new approach to the measurement of bivariate inequality. Int $J$ Environ Res Public Health 2017;14:673. 